\title{
ON NUMERICAL RANGES OF GENERALIZED DERIVATIONS AND RELATED PROPERTIES
}

\author{
SEN-YEN SHAW \\ (Received 28 February 1982; revised 11 November 1982) \\ Communicated by G. Brown
}

\begin{abstract}
This paper is concerned with the numerical range and some related properties of the operator $\Delta \mid S$ : $T \rightarrow A T-T B(T \in \delta)$, where $A, B$ are (bounded linear) operators on the normed linear spaces $X$ and $\mathbf{Y}$, respectively, and $\mathcal{S}$ is a linear subspace of the space $\mathcal{L}(\mathbf{Y}, \mathbf{X})$ of all operators from $\mathbf{Y}$ to $\mathbf{X} \mathcal{S}$ is assumed to contain all finite operators, to be invariant under $\Delta$, and to be suitably normed (not necessarily with the operator norm). Then the algebra numerical range of $\Delta \mid \delta$ is equal to the difference of the algebra numerical ranges of $A$ and $B$. When $\mathbf{X}=\mathbf{Y}$ and $\delta=\mathcal{L}(\mathbf{X}), \Delta$ is Hermitian (resp. normal) in $\mathscr{L}(\mathcal{L}(\mathbf{X}))$ if and only if $A-\lambda$ and $B-\lambda$ are Hermitian (resp. normal) in $\mathcal{L}(\mathbf{X})$ for some scalar $\lambda$; if $\mathbf{X}:=\mathbf{H}$ is a Hilbert space and if $S$ is a $C^{*}$-algebra or a minimal norm ideal in $\mathcal{L}(\mathbf{H})$, then any Hermitian (resp. normal) operator on $S$ is of the form $\Delta / \delta$ for some Hermitian (resp. normal) operators $A$ and $B . A T=T B$ implies $A^{*} T=T B^{*}$, provided that $A$ and $B^{*}$ are hyponormal operators on the Hilbert spaces $H_{1}$ and $\mathbf{H}_{2}$, respectively, and $T$ is a Hilbert-Schmidt operator from $\mathbf{H}_{2}$ to $\mathbf{H}_{1}$.
\end{abstract}

1980 Mathematics subject classification (Amer. Math. Soc.): 47 A 12, 47 B 15, 47 B 47.

Keywords and phrases: algebra numerical range, derivation, Hermitian and normal operators, hyponormal operator, normed operator ideal, Schatten $p$-class, $C^{*}$-algebra, Hilbert-Schmidt operator.

\section{Introduction}

Let $\mathbf{X}$ be a complex normed linear space and let $\mathfrak{L}(\mathbf{X})$ be the algebra of all (bounded linear) operators on $\mathbf{X}$. The spatial numerical range of an operator $A$ on $\mathbf{X}$ is given by $W(A ; \mathfrak{L}(\mathbf{X})):=\{f(A x) ;(x, f) \in \pi(\mathbf{X})\}$, where $\pi(\mathbf{X})$ denotes the set of all pairs $(x, f) \in \mathbf{X} \times \mathbf{X}^{\prime}$ such that $\|x\|=\|f\|=f(x)=1$. The algebra numerical range of $A$ in $\mathcal{L}(\mathbf{X})$ is given by $V(A ; \mathfrak{L}(\mathbf{X})):=\{F(A) ;(I, F) \in$ $\pi(\mathscr{L}(\mathbf{X}))\}$, where $I$ is the identity operator. It is known that $V(A ; \mathcal{L}(\mathbf{X}))$ is compact

(C) 1984 Australian Mathematical Society $0263-6115 / 84 \$ A 2.00+0.00$ 
and is the closed convex hull of $W(A ; \mathcal{L}(\mathbf{X}))$. When $\mathbf{X}=\mathbf{H}$ is a Hilbert space with inner product $\langle\rangle,, W(A ; \mathcal{L}(\mathbf{X}))$ reduces to the classical numerical range $W(A):=\{\langle A x, x\rangle ; x \in \mathbf{H},\|x\|=1\}$ (see [4] for details).

If $B$ is an operator on another normed linear space $Y$, then the generalized derivation $\Delta=\Delta_{A, B}$, defined by $\Delta(T):=A T-T B$, is an operator on the space $\mathcal{L}(\mathbf{Y}, \mathbf{X})$ of all operators from $\mathbf{Y}$ to $\mathbf{X}$. In this paper, we consider the numerical ranges of restrictions of $\Delta$ to certain invariant subspaces $\delta$ and some consequences. First, the algebra numerical range of $\Delta \mid \mathfrak{S}$ is shown to be the difference of $V(A ; \mathcal{L}(\mathbf{X}))$ and $V(B ; \mathcal{L}(\mathbf{Y}))$, provided that $\delta$ contains all finite-rank operators and is suitably normed. Then it is applied to determine when $\Delta$ or $\Delta \mid \delta$ is Hermitian or normal, and to derive a Fuglede-Putnam theorem for hyponormal operators. The results will extend some theorems of Kyle [8], Sourour [13] and Berberian [3], respectively.

\section{The numerical range}

We will assume that $\delta$ is a linear subspace of $\mathscr{E}(\mathbf{Y}, \mathbf{X})$ equipped with a norm ||$|\cdot|||$ (possibly different from the operator norm $\|\cdot\|$ ) such that the following conditions are satisfied:

(1) $A \mathcal{S} \subset \mathcal{S}$ and $\delta B \subset \mathcal{S}$; (2) If $D \in \mathcal{E}(\mathbf{X}), T \in \mathcal{S}, E \in \mathcal{L}(\mathbf{Y})$ and $D T E \in \mathcal{S}$, then $\||D T E|\| \leqslant\|D\|\|\mid\| T\|\|\|E\| ;(3)\|T\| \leqslant\|T\|$ for all $T$ in $\mathcal{S}$, and the equality holds whenever $T$ has rank one; (4) $\mathfrak{S}$ contains all finite rank operators from $Y$ to $\mathbf{X}$.

It follows from (1) that $\delta$ is an invariant subspace of $\Delta=\Delta_{A, B}$, and from (2) that the restriction $\Delta \mid \delta$ of $\Delta$ is a bounded linear operator on $(\mathcal{S},\||\cdot|\|)$. We consider the numerical range of $\Delta \mid \delta$.

THEOREM 1.1. For operators $A \in \mathcal{L}(\mathbf{X})$ and $B \in \mathcal{C}(\mathbf{Y})$ let $(\delta,\|\mid \cdot\|)$ be the normed linear space as mentioned above. Then

$$
V(\Delta \mid \mathcal{S} ; \mathcal{L}(\S))=V(A ; \mathcal{L}(\mathbf{X}))-V(B ; \mathcal{L}(\mathbf{Y})) .
$$

Proof. We first prove that the left side is contained in the right side. So, let $\lambda$ be an arbitrary element of $V(\Delta \mid \mathcal{S} ; \mathcal{L}(\delta))$. Then $\lambda=f(\Delta \mid \delta)$ for some $f \in(\mathcal{L}(\delta))^{\prime}$ such that $\|f\|=f\left(I_{\mathfrak{R}(\delta)}\right)=1$, where $I_{\mathfrak{L}(\delta)}$ denotes the identity operator in $\mathcal{L}(\mathcal{L})$. It is clear that the set $\mathscr{N}$ (resp. $\mathscr{T}$ ) of all $D \in \mathcal{L}(\mathbf{X})$ (resp. $E \in \mathcal{L}(\mathbf{Y})$ ) such that $D \delta \subset \delta($ resp. $\delta E \subset \delta)$ is a linear subspace of $\mathscr{L}(\mathbf{X})$ (resp. $\mathscr{L}(\mathbf{Y})$ ) containing $I_{\mathfrak{E}(\mathbf{X})}$ and $A$ (resp. $I_{\mathfrak{L}(Y)}$ and $B$ ). Define a linear functional $F$ on $\mathfrak{N}$ by $F(D)=f\left(L_{D} \mid \S\right)$ $(D \in \mathscr{R})$ and a linear functional $G$ on $\Re$ by $G(E)=f\left(R_{E} \mid \delta\right)(E \in \mathfrak{X})$, where $L_{D}$ and $R_{E}$ stand for the left multiplication by $D$ and the right multiplication by 
$E$, respectively. Now the Hahn-Banach theorem guarantees the existence of $\hat{F}$ in $(\mathfrak{E}(\mathbf{X}))^{\prime}$ and $\hat{G}$ in $(\mathscr{L}(\mathbf{Y}))^{\prime}$ such that $\hat{F}|\Re=F,\|\hat{F}\|=\|F\|, \hat{G}| \Re=G$ and $\|\hat{G}\|=$ $\|G\|$. Since $\hat{F}\left(I_{\mathfrak{L}(\mathbf{X})}\right)=F\left(I_{\mathfrak{R}(\mathbf{X})}\right)=f\left(I_{\mathfrak{L}(\mathcal{S})}\right)=1$ and since $|F(D)| \leqslant\|f\|\left\|L_{D} \mid \delta\right\|=$ $\sup \{|||D T\||; T \in \mathcal{S}|\| T \||=1\} \leqslant,\|D\|$ for all $D$ in $\mathscr{R}$, we have that $\|\hat{F}\|=\|F\|$ $\leqslant 1=\hat{F}\left(I_{\mathfrak{E}(\mathbf{X})}\right) \leqslant\|\hat{F}\|$, that is, $\left(I_{\mathfrak{L}(\mathbf{X})}, \hat{F}\right)$ belongs to $\pi(\mathfrak{L}(\mathbf{X}))$. Similarly, we have $\left(I_{\mathcal{E}(\mathbf{Y})}, \hat{G}\right) \in \pi(\mathcal{E}(\mathbf{Y}))$. Hence

$$
\begin{aligned}
\lambda & =f(\Delta \mid \delta)=f\left(L_{A} \mid \delta\right)-f\left(R_{B} \mid \delta\right) \\
& =F(A)-G(B)=\hat{F}(A)-\hat{G}(B) \\
& \in V(A ; \mathcal{L}(\mathbf{X}))-V(B ; \mathcal{L}(\mathbf{Y})) .
\end{aligned}
$$

To prove the other inclusion, it suffices to show that

$$
V(\Delta \mid S ; \mathfrak{L}(\mathfrak{S})) \supset W(A ; \mathfrak{L}(\mathbf{X}))-W(B ; \mathfrak{L}(\mathbf{Y}))
$$

since the closed convex hull of the set on the right side is $V(A ; \mathscr{L}(\mathbf{X}))-$ $V(B ; \mathfrak{L}(\mathbf{Y}))$, by an elementary proof. So, let $\alpha=g(A x)$ with $(x, g) \in \pi(\mathbf{X})$ and let $\beta=h(B y)$ with $(y, h) \in \pi(\mathbf{Y})$. Using the usual notation $x \otimes h$ for the rank-one operator: $z \rightarrow h(z) x(z \in \mathbf{Y})$, we define the linear functional $P$ on $\mathcal{L}(\mathcal{S})$ by

$$
P(\Omega):=g([\Omega(x \otimes h)] y)(\Omega \in \mathcal{L}(\mathcal{S})) .
$$

Clearly we have $P\left(I_{\mathcal{R}(\S)}\right)=1$ and, by (3) and (4),

$$
\begin{aligned}
|P(\Omega)| & \leqslant\|g\|\|\Omega(x \otimes h)\|\|y\| \leqslant\|\| \Omega(x \otimes h)\|\| \leqslant\|\Omega\|\|\| x \otimes h\|\| \\
& =\|\Omega\|\|x \otimes h\|=\|\Omega\|,
\end{aligned}
$$

that is, $\left(I_{\mathcal{E}(\delta)}, P\right) \in \pi(\mathscr{L}(\delta))$. Hence $V(\Delta \mid \delta ; \mathcal{L}(\delta))$ contains the number

$$
\begin{aligned}
P(\Delta \mid \delta) & =g(A(x \otimes h) y-(x \otimes h) B y) \\
& =g(A x) h(y)-g(x) h(B y)=\alpha-\beta .
\end{aligned}
$$

The proof is complete.

REMark. Conditions (3) and (4) are used only in proving the direction " $\supset$ ", therefore the inclusion " $\subset$ " will hold for any subspace satisfying (1) and (2). That (4) is essential for the direction " $\supset$ " is easily seen from the example where $\mathbf{X}=\mathbf{Y}=C^{2}, A=B=\left(\begin{array}{ll}0 & 0 \\ 1 & 0\end{array}\right), \mathfrak{S}=\operatorname{span}\{A\}$ and $\|\cdot \cdot\|=\|\cdot\|$.

The following are some examples of qualified subspaces $(\mathcal{S},|||\cdot| \mid)$ ):

(a) Components in $\mathscr{E}(\mathbf{Y}, \mathbf{X})$ of all the operator ideals on Banach spaces, as studied in Chapter 1 of [9], such as the classes of finite operators, approximate operators, compact operators, weakly compact operators, completely continuous 
operators, unconditionally summing operators, separable operators, Kato operators and Pelczynski operators.

(b) Components in $\mathcal{L}(\mathbf{Y}, \mathbf{X})$ of all the normed operator ideals on Banach spaces, as studied in Chapter 6 of [9], such as nuclear operators, integral operators, absolutely summing operators and Hilbert operators. (Compare the assumptions on $(\mathcal{S},|||\cdot| \mid)$ with Definitions 6.1.1, 6.2.2 and Propositions 6.1.4, 6.1.5 of [9].)

(c) The Schatten $p$-class $C_{p}\left(\mathbf{H}_{2}, \mathbf{H}_{1}\right)(1 \leqslant p<\infty)$ or approximable operators from a Hilbert space $\mathbf{H}_{2}$ to another Hilbert space $\mathbf{H}_{1}$, that is, operators $T$ such that $\|T\|_{p}:=\left[\operatorname{trace}\left(T^{*} T\right)^{p / 2}\right]^{1 / p}<\infty$, (see [9, page 216]). In the case where $p=1$ these are the operators of trace class (nuclear operators), and $p=2$ yields the Hilbert space of Hilbert-Schmidt operators (see [2, Chapter 12]).

Corollary 1.2. For any $A \in \mathfrak{L}(\mathbf{X})$ and $B \in \mathcal{L}(\mathbf{Y})$,

$$
V(\Delta ; \mathscr{L}(\mathscr{L}(\mathbf{Y}, \mathbf{X})))=V(A ; \mathscr{L}(\mathbf{X}))-V(B ; \mathscr{L}(\mathbf{Y})) .
$$

This contains Kyle's result [8] (for the case $\mathbf{X}=\mathbf{Y}$ ) as a special case.

Corollary 1.3. Let $A$ and $B$ be any operators on Hilbert spaces $\mathbf{H}_{1}$ and $\mathbf{H}_{2}$, respectively, and let $C_{p}\left(\mathbf{H}_{2}, \mathbf{H}_{1}\right)$ be normed with $\|\cdot\|$ or $\|\cdot\|_{p}$. Then

$$
V\left(\Delta \mid C_{p}\left(\mathbf{H}_{2}, \mathbf{H}_{1}\right) ; \mathfrak{e}\left(C_{p}\left(\mathbf{H}_{2}, \mathbf{H}_{1}\right)\right)\right)=W(A)^{-}-W(B)^{-} .
$$

Thus Corollary 1.3 becomes a numerical range analogue of Fialkow's [5] formula for spectra: $\sigma\left(\Delta \mid C_{p}(\mathbf{H})\right)=\sigma(A)-\sigma(B)$.

We end this section by deriving from Theorem 1.1 the following known property, which will be of use in Section 2.

COROLlary 1.4. If $A T=T B$ holds for all rank-one operators $T$ in $\mathcal{L}(\mathbf{Y}, \mathbf{X})$, then $A=\lambda I_{\mathfrak{L}(\mathbf{X})}$ and $B=\lambda I_{\mathfrak{L}(\mathbf{Y})}$ for some scalar $\lambda$.

Proof. Take $\delta$ to be the space of all finite rank operators. Then $\Delta \mid \delta=0$ and so $V(A ; \mathfrak{L}(\mathbf{X}))-V(B ; \mathfrak{L}(\mathbf{Y}))=V(\Delta \mid \mathfrak{L}(\Sigma))=\{0\}$, or equivalently, $V(A ; \mathfrak{L}(\mathbf{X}))$ $=V(B ; \mathcal{L}(\mathbf{Y}))=\{\lambda\}$ for some scalar $\lambda$. It follows that $V\left(A-\lambda I_{\mathcal{E}(\mathbf{X})} ; \mathcal{L}(\mathbf{X})\right)=\{0\}$ and

$$
\left\|A-\lambda I_{\mathfrak{L}(\mathbf{X})}\right\| \leqslant e \max \left\{|\mu| ; \mu \in V\left(A-\lambda I_{\mathfrak{L}(\mathbf{X})} ; \mathcal{L}(\mathbf{X})\right)\right\}=0
$$

(see [4, page 34]). Hence $A=\lambda I_{\mathfrak{E}(\mathbf{X})}$, and similarly $B=\lambda I_{\mathfrak{L}(\mathbf{Y})}$. 


\section{Hermitian and normal derivations}

An operator $A$ on a normed linear space $\mathbf{X}$ is said to be Hermitian if its numerical range is contained in the real line and it is normal if $A=H+i K$ for some commuting Hermitian operators $H$ and $K$. In this section we try to answer partly the question about when the operator $\Delta / \delta$ is Hermitian or normal.

First, from the formula (*) comes immediately the following

CoRollary 2.1. Let $(\mathcal{S},\|\cdot \mid\|)$ be as assumed in Theorem 1.1. Then $\Delta \mid \mathcal{S}$ is Hermitian in $\mathcal{L}(\mathcal{L}(\mathbf{Y}$,$) if and only if A-\lambda I \in \mathcal{L}(\mathbf{X})$ and $B-\lambda I \in \mathcal{Q}(\mathbf{Y})$ are Hermitian for some scalar $\lambda$.

Kyle [8] has proved that when $\mathbf{X}=\mathbf{Y}$ is a Banach space and when $A=B, \Delta$ is normal if and only if $A$ is normal in $\mathcal{E}(\mathbf{X})$. We shall extend this result under various situations. The statement for the most general situation is as follows.

Theorem 2.2. Let $A \in \mathcal{L}(\mathbf{X})$ and $B \in \mathcal{L}(\mathbf{Y})$ be of the forms $A=H+i K$ and $B=M+i N$, where $H, K, M$ and $N$ are Hermitian operators. Suppose that $(\mathcal{S},\||\cdot|\|)$ satisfies $H \varsigma \subset \delta, K \delta \subset \delta, \delta M \subset \delta, \delta N \subset \delta$ and conditions (2), (3), (4). Then $\Delta \mid \Sigma$ is normal if and only if both $A$ and $B$ are normal.

Proof. $\Delta_{A, B}$ can be written as $\Delta_{H, M}+i \Delta_{K, N}$, where $\Delta_{H, M}$ and $\Delta_{K, N}$ and their restrictions to $S$ are Hermitian, by Corollary 2.1. Now, from the easily verified identity

$$
\Delta_{H, M} \Delta_{K, N}-\Delta_{K, N} \Delta_{H, M}=\Delta_{H K-K H, M N-N M}
$$

we see that $\Delta / \delta$ is normal if and only if $(H K-K H) T=T(M N-N M)$ for all $T$ in $\mathcal{S}$. Since $\mathfrak{S}$ contains all finite rank operators, the latter condition is, by Corollary 1.4, equivalent to that $H K-K H=\lambda I_{\mathfrak{L}(\mathbf{X})}$ and $M N-N M=\lambda I_{\mathfrak{L}(\mathbf{Y})}$ for some scalar $\lambda$. But this is possible only when $\lambda=0$, that is, $A$ and $B$ are normal (see [10, page 332]).

It follows that for $\Delta / \mathcal{S}$ to be normal it is sufficient that $A$ and $B$ are normal. That this is also necessary in case $\mathbf{X}$ and $\mathbf{Y}$ are Hilbert spaces is already contained. in the above theorem.

Corollary 2.3. Let $\mathbf{H}_{1}$ and $\mathbf{H}_{2}$ be Hilbert spaces. For $A \in \mathcal{E}\left(\mathbf{H}_{1}\right)$ and $B \in$ $\mathcal{E}\left(\mathbf{H}_{2}\right)$ let $\delta \subset \mathcal{L}\left(\mathbf{H}_{2}, \mathbf{H}_{1}\right)$ be a subspace satisfying conditions (1)-(4) (for example, $C_{p}\left(\mathbf{H}_{2}, \mathbf{H}_{1}\right)$ with norm $\|\cdot\|_{p}$ or operator norm $\left.\|\cdot\|\right)$. Then $\Delta \mid \delta$ is normal if and only if both $A$ and $B$ are normal. 
Remark. When $A$ and $B$ are normal operators on a Hilbert space $\mathbf{H}, \Delta$ and $\Delta \mid C_{2}(\mathbf{H})$ become normal operators on the Banach space $\mathcal{L}(\mathbf{H})$ and the Hilbert space $\left(C_{2}(\mathbf{H}),\|\cdot\|_{2}\right)$, respectively. It follows (see [6, Theorem $\left.\mathrm{A}\right]$ or [1]) that the null space $N(\Delta)$ is orthogonal to the range $R(\Delta)$ of $\Delta$. Hence we have

$$
R(\Delta)^{-} \oplus N(\Delta) \supset\left(R\left(\Delta \mid C_{2}(\mathbf{H})\right)^{=} \oplus N\left(\Delta \mid C_{2}(\mathbf{H})\right)\right)^{-}=C_{2}(\mathbf{H})^{-},
$$

where the superscripts " $=$ " and " $=$ " denote the closure relative to $\|\cdot\|$ and $\|\cdot\|_{2}$, respectively. Thus $R(\Delta)^{-} \oplus N(\Delta)$ contains all compact operators while it is in general strictly less than $\mathscr{L}(\mathbf{H})[1]$.

Since a general operator on a normed linear space is not necessarily of the form $H+I K$, with $H$ and $K$ Hermitian, it is not known from Theorem 2.2 whether a normal $\Delta_{A, B} \mid \delta(A \in \mathcal{L}(\mathbf{X}), B \in \mathcal{L}(\mathbf{Y}))$ must be made of two normal $A$ and $B$. But, at least when $\mathbf{X}$ is equal to $\mathbf{Y}$ and when $\mathcal{S}=\mathscr{L}(\mathbf{X})$, this is true, as is shown by the following extension of Kyle's result.

THEOREM 2.4. Let $A$ and $B$ be operators on a normed linear space $X$. Then $\Delta_{A, B}$ is normal in $\mathcal{L}(\mathcal{E}(\mathbf{X}))$ if and only if both $A$ and $B$ are normal in $\mathcal{E}(\mathbf{X})$.

This will follow from Theorem 2.2 (with $\delta=\mathfrak{L}(\mathbf{X})$ ) and the next

LEMMA 2.5. $\Delta_{A, B}=\Phi+i \Psi$ for some Hermitian operators $\Phi$ and $\Psi$ in $\mathcal{L}(\mathcal{L}(\mathbf{X}))$ if and only if $A=H+i K$ and $B=M+i N$ for some Hermitian operators $H, K, M$ and $N$ in $\mathcal{L}(\mathbf{X})$.

Proof. Suppose $\Delta=\Phi+i \Psi$ where $\Phi$ and $\Psi$ are Hermitian. Fix a pair $\left(x_{0}, f\right)$ in $\pi(\mathbf{X})$ and define operators $H_{1}, K_{1}, M_{1}$ and $N_{1}$ by $H_{1} x:=(\Phi(x \otimes f)) x_{0}$, $K_{1} x:=(\Psi(x \otimes f)) x_{0}(x \in X), M_{1}:=H_{1}-\Phi(I)$ and $N_{1}:=K_{1}-\Psi(I)$, respectively, where $I$ is the identity operator on $\mathbf{X}$.

We first show that these operators are Hermitian. To show that $H_{1}$ is Hermitian, we will prove that $g\left(H_{1} x\right)$ is real for any pair $(x, g)$ in $\pi(\mathbf{X})$. Indeed, for a fixed $(x, g)$ in $\pi(\mathbf{X})$ there corresponds the linear functional $G: T \rightarrow g\left(T x_{0}\right)$ $(T \in \mathcal{L}(\mathbf{X}))$ on $\mathcal{L}(\mathbf{X})$ which satisfies: $\|G\|=G(x \otimes f)=\|x \otimes f\|=1$, that is, $(x \otimes f, G) \in \pi(\Lambda(\mathbf{X}))$. This implies that

$$
g\left(H_{1} x\right)=g\left((\Phi(x \otimes f)) x_{0}\right)=G(\Phi(x \otimes f)) \in W(\Phi ; \mathcal{L}(\mathscr{L}(\mathbf{X}))) \subset R .
$$

By a similar way one can show that $K_{1}$ is also Hermitian. To claim that $\Phi(I)$ is Hermitian we observe that $F(\Phi(I))$ belongs to $W(\Phi, \mathcal{L}(\mathcal{L}(\mathbf{X})))$ for every $(I, F)$ in $\pi(\mathscr{L}(\mathbf{X}))$, or equivalently, $V(\Phi(I) ; \mathscr{L}(\mathbf{X})) \subset(\Phi ; \mathcal{L}(\mathscr{L}(\mathbf{X}))) \subset R$. Similarly, $\Psi(I)$ is Hermitian. 
Now we have, for $D \in \mathfrak{L}(\mathbf{X})$ and $x \in \mathbf{X}$,

$$
\begin{aligned}
\left(\Delta_{H_{1}, M_{1}}(D)\right) x & =\left(H_{1} D-D M_{1}\right) x=H_{1} D x-D H_{1} x+D \Phi(I) x \\
& =(\Phi(D x \otimes f)) x_{0}-D(\Phi(x \otimes f)) x_{0}+D \Phi(I) x,
\end{aligned}
$$

and similarly

$$
\left(\Delta_{K_{1}, N_{1}}(D)\right) x=(\Psi(D x \otimes f)) x_{0}-D(\Psi(x \otimes f)) x_{0}+D \Psi(I) x .
$$

Thus

$$
\begin{aligned}
&\left(\left(\Delta_{H_{1}, M_{1}}+i \Delta_{K_{1}, N_{1}}\right) D\right) x \\
&=\left(\Delta_{A, B}(D x \otimes f)\right) x_{0}-D\left(\Delta_{A, B}(x \otimes f)\right) x_{0}+D(A-B) x \\
&= A(D x \otimes f) x_{0}-(D x \otimes f) B x_{0} \\
&-D(A(x \otimes f)-(x \otimes f) B) x_{0}+D(A-B) x \\
&= A D x-D A x+D(A-B) x \\
&= \Delta_{A, B}(D) x .
\end{aligned}
$$

That is, $\left(A-H_{1}-i K_{1}\right) D=D\left(B-M_{1}-i N_{1}\right)$ holds for every $D$ in $\mathscr{L}(\mathbf{X})$. It follows from Corollary 1.4 that $A=H_{1}+i K_{1}+\lambda I$ and $B=M_{1}+i N_{1}+\lambda I$ for some scalar $\lambda$. Now we can take $H=H_{1}+(\operatorname{Re} \lambda) I, K=K_{1}+(\operatorname{Im} \lambda) I, M=M_{1}$ $+(\operatorname{Re} \lambda) I$ and $N=N_{1}+(\operatorname{Im} \lambda) I$ as the desired Hermitian operators.

So far, the question about when $\Delta_{A, B} \mid \delta$ is Hermitian in $\mathcal{L}(\mathcal{S})(\mathcal{S} \subset \mathcal{L}(\mathbf{Y}, \mathbf{X}))$ has been answered by Corollary 2.1 , and the question about when $\Delta \mid \delta$ is normal has been answered by Theorem 2.2 for special operators $A$ and $B$ on normed linear spaces $\mathbf{X}$ and $\mathbf{Y}$, by Corollary 2.3 for the case where $\mathbf{X}$ and $\mathbf{Y}$ are Hilbert spaces, and by Theorem 2.4 for the case where $X$ is the same normed linear space as $Y$ and $\delta$ is $\mathcal{L}(\mathbf{X})$. But the latter question for the more general case where $\mathbf{X} \neq \mathbf{Y}$ or where $\mathbf{X}=\mathbf{Y}$ and $\mathcal{S} \neq \mathfrak{L}(\mathbf{X})$ remain unanswered. It is unknown whether there exist nonnormal operators $A, B$ such that $\Delta_{A, B} \mid \delta$ is normal.

On the other hand, when $\mathbf{X}=\mathbf{Y}=\mathbf{H}$ is a Hilbert space, one can deduce a stronger result than Corollaries 2.1 and 2.3. Indeed, a result of Sinclair [12, page 213] states that a Hermitian operator on a $C^{*}$-algebra (with identity) is the sum of a left multiplication by a Hermitian element in the algebra and a *-derivation, and a result of Kadison [7] and Sakai [11] asserts that every derivation of a $C^{*}$-algebra acting on $H$ is spatial (that is, of the form $\Delta_{A, A}$, with $A$ and element in the weak operator closure of the algebra). These facts together with Corollary 2.1 imply that an operator on a $C^{*}$-algebra $S$ in $\mathscr{L}(\mathbf{H})$ is Hermitian if and only if it is of the form $\Delta_{H, M} \mid \delta$ for some Hermitian operators $H$ and $M$ (in the weak operator closure of $\Im$ ). Recently, Sourour [13] has proved the same assertion for the case where $S$ is a minimal norm ideal (including the $C_{p}(\mathbf{H})$ ideals, $p \neq 2$ ). Thus every 
normal operator on such $\delta$ has to be of the form $\Delta_{A, B} \mid \delta$, with $A=H+i K$ and $B=M+i N$ for some Hermitian $H, K, M$ and $N$. Using Theorem 2.2 we obtain the following

TheOREM 2.6. Let $S$ be a minimal norm ideal or a $C^{*}$-algebra in $\mathcal{L}(\mathbf{H})$, which contains all finite rank operators. Then an operator $U$ on $\delta$ is Hermitian (resp. normal) in $\mathcal{L}(\delta)$ if and only if $U=\Delta_{A, B} \mid \delta$ for some Hermitian (resp. normal) operators $A$ and $B$.

\section{Berberian's theorem}

As another application of Theorem 1.1, we shall derive an extension of the Fuglede-Putnam theorem to hyponormal operators $A$ and $B^{*}$ on Hilbert spaces $\mathbf{H}_{1}$ and $\mathbf{H}_{2}$, respectively. It is also a slight extension of a theorem of Berberian [3] who proved in a different way the special case where $\mathbf{H}_{1}=\mathbf{H}_{2}$.

A natural and consistent definition for a hyponormal operator $A$ on a normed linear space $\mathbf{X}$ is that it can be written as $A=H+i K$ for some Hermitian operators $H$ and $K$ such that $A^{*} A-A A^{*}=2 i(H K-K H)$ is positive (that is, has nonnegative numerical range). Suppose $B^{*}=M-i N$ is a hyponormal operator on another normed linear space $Y$. Then the operator $\Delta_{A, B} \mid \delta$ is also hyponormal. Indeed, from the easily verified identity:

$$
\begin{aligned}
\Delta^{*} \Delta-\Delta \Delta^{*} & =2 i\left(\Delta_{H, M} \Delta_{K, N}-\Delta_{K, N} \Delta_{H, M}\right)=2 i \Delta_{H K-K H, M N-N M} \\
& =\Delta_{A^{*} A-A A^{*}, B^{*} B-B B^{*}}
\end{aligned}
$$

we see that the numerical range of $(\Delta \mid \S)^{*}(\Delta \mid \S)-(\Delta \mid \S)(\Delta \mid \S)^{*}$, as the sum of the numerical ranges of the two positive operators $A^{*} A-A A^{*}$ and $B B^{*}-B^{*} B$ is nonnegative.

In particular, if $\mathbf{X}=\mathbf{H}_{1}$ and $\mathbf{Y}=\mathbf{H}_{2}$ are two Hilbert spaces, then $\Delta \mid C_{2}\left(\mathbf{H}_{2}, \mathbf{H}_{1}\right)$ is a hyponormal operator on the Hilbert space $\left(C_{2}\left(\mathbf{H}_{2}, \mathbf{H}_{1}\right),\|\cdot\|_{2}\right)$. Hence we have $\|\Delta T\|_{2} \geqslant\left\|\Delta^{*} T\right\|_{2}$ for all $T$ in $C_{2}\left(\mathbf{H}_{2}, \mathbf{H}_{1}\right)$. Since $\left(\Delta_{A, B}\right)^{*}=\Delta_{A^{*}, B^{*}}$, we have proved the following

THEOREM 3.1. Let $A$ and $B^{*}$ be hyponormal operators on the Hilbert spaces $\mathbf{H}_{1}$ and $\mathbf{H}_{2}$, respectively. If $T$ is a Hilbert-Schmidt operator from $\mathbf{H}_{2}$ to $\mathbf{H}_{1}$ such that $A T=T B$, then $A^{*} T=T B^{*}$. 


\section{References}

[1] J. Anderson and C. Foias, 'Properties which normal operators share with normal derivations and related operators', Pacific J. Math. 61 (1975), 313-325.

[2] J. P. Aubin, Applied functional analysis (Wiley-Interscience, New York, 1979).

[3] S. K. Berberian, 'Extensions of a theorem of Fuglede and Putnam', Proc. Amer. Math. Soc. 71 (1978), 113-114.

[4] F. F. Bonsall and J. Duncan, Numerical ranges of operators on normed spaces and of elements of normed algebras (London Math. Soc. Lecture Note Series 2, Cambridge Univ. Press, 1971).

[5] L. Fialkow, 'A note on the operator $X \rightarrow A X-X B$ ', Trans. Amer. Math. Soc. 243 (1978), 147-168.

[6] C. K. Fong, 'Normal operators on Banach spaces', Glasgow Math. J. 20 (1979), 163-168.

[7] R. V. Kadison, 'Derivations of operator algebras', Ann. of Math. 83 (1966), 280-293.

[8] J. Kyle, 'Numerical ranges of derivations', Proc. Edinburgh Math. Soc. 21 (1978), 33-39.

[9] A. Pietsch, Operator ideals (North-Holland Mathematical library vol. 20, 1980).

[10] W. Rudin, Functional analysis (McGraw-Hill, New York, 1973).

[11] S. Sakai, 'Derivations of $W^{*}$-algebras', Ann. of Math. 83 (1966), 273-279.

[12] A. M. Sinclair, 'Jordan homomorphisms and derivations on semi-simple Banach algebras', Proc. Amer. Math. Soc. 24 (1970), 209-214.

[13] A. R. Sourour, 'Isometries of norm ideals of compact operators', preprint.

\section{Department of Mathematics}

National Central University

Chung-Li, Taiwan 320

Republic of China 